\title{
Una clase sobre los usos de los verbos habituales basada en un índice marcador de la dificultad de aprendizaje
}

\author{
RYO TSUTAHARA \\ Universidad de Kyushu \\ tsutahara@flc.kyushu-u.ac.jp
}

\begin{abstract}
Resumen: En este trabajo presentamos nuestra experiencia de enseñanza de la polisemia y usos de los verbos transitivos más habituales, utilizando como referencia un índice que señala la dificultad de aprendizaje de cada verbo. Consideramos que enseñar las palabras más frecuentes es sumamente importante, ya que según nuestro análisis el $70 \%$ del discurso de los nativos lo ocupan apenas 600 palabras. El índice que proponemos calcula la cantidad de tipos semánticos de objetos frecuentes de cada verbo, el número de coocurrencias de cada verbo con aquellos objetos y el total de verbos japoneses que se corresponden semánticamente con ellos. El objetivo es impartir clases de manera eficiente, puesto que organizamos las lecciones y elaboramos sus materiales tomando como referencia la información que aporta el índice, el cual nos sirve para establecer qué verbos deben tratarse con mayor cuidado y esfuerzo.
\end{abstract}

Palabras clave: verbos, polisemia, colocaciones, dificultad de aprendizaje.

\section{A class on the uses of the usual verbs based on a labeling index of the difficulty of learning}

\begin{abstract}
In this paper, we present our experience of teaching polysemy and uses of the most common transitive verbs, using an index as a reference that points out the difficulty of learning each verb. We believe that teaching the most frequent words is extremely important, since according to the analysis $70 \%$ of the speech of the natives occupy only 600 words. The index that we propose calculates the number of semantic types of frequent objects of each verb, the number of co-occurrences of each verb with those objects and the total of Japanese verbs that correspond semantically with them. The objective is to teach classes efficiently, since we organize the lessons and elaborate their materials taking as reference the information provided by the index, which helps us to establish which verbs should be treated with the greatest care and effort.
\end{abstract}

Key words: verbs, polysemy, collocations, difficulty of learning.

\section{Introducción}

En este trabajo presentamos nuestra experiencia de enseñar a estudiantes japoneses la polisemia y usos de los verbos transitivos más habituales del español, basándonos en un índice que señala la dificultad de aprendizaje de cada verbo. Para impartir clases de manera eficiente, desarrollamos un método para medir la dificultad léxica y puntuamos los verbos transitivos que planeamos tratar en la clase. Teniendo en cuenta el resultado de la evaluación, especificamos cuáles son los verbos que requieren una explicación más detallada y cuáles son las perspectivas que deben enfatizarse en el aula. La novedad 
de esta experiencia radica en el empleo de este índice, por lo que prestaremos particular atención a las ventajas que comporta su aplicación.

\subsection{Enseñar polisemia y uso de los verbos más frecuentes}

Ampliar el vocabulario es tan importante como adquirir el conocimiento de la gramática, practicar la conversación, etc. Particularmente, consideramos imprescindible manejar las palabras más frecuentes o básicas. Según nuestro análisis del corpus, alrededor del $70 \%$ del discurso de los nativos emplea únicamente las 600 palabras más frecuentes. El hecho de que la mayoría de estas palabras sean altamente polisémicas contribuye a ello, de ahí que profundizar en el conocimiento de los términos más frecuentes resulte práctico y eficiente para mejorar la capacidad lingüística.

No obstante, desde el punto de vista de los estudiantes, tal abundancia de significados y usos, a veces muy complicados, requiere de explicaciones bien detalladas.

Asumiendo esta situación, impartimos clases sobre la polisemia y usos múltiples de los 50 verbos transitivos más frecuentes. En las clases, para enseñarlos, tratamos las colocaciones de tipo " $\mathrm{N}+\mathrm{V}$ " $\mathrm{y}$ "V $+\mathrm{N}$ ", en vez de presentar cada palabra de forma independiente y descontextualizada. Hablar de las colocaciones, nos da la oportunidad de enseñar características semánticas de las diversas palabras que las constituyen ${ }^{1}$. Además, también nos permite enfatizar el hecho de que el significado de las palabras depende de sus coapariciones. Mostrárselo a los estudiantes es enseñar el mecanismo de la polisemia, es decir, profundizar el entendimiento del léxico de los estudiantes. La otra ventaja de tratar colocaciones es poder enseñar la manera espontánea de combinar las palabras. Conseguir esta competencia es importante para manejar un idioma extranjero, por lo que consideramos que esta es una manera eficaz de enseñar el vocabulario ${ }^{2}$.

Nuestros estudiantes llevan unos seis meses aprendiendo español y el curso es opcional para los que intentan llegar al nivel A2 en un año. La mayoría de ellos se están especializando en estudios de letras y ciencias sociales. Las actividades que desarrollamos en esas clases las presentaremos en la sección 4.

A la hora de planificar el curso, lo que discutimos más fue cómo distribuir el tiempo y esfuerzo en las clases. El periodo docente ocupó 14 sesiones, con una duración de 90 minutos, durante el semestre de otoño. En tan limitado periodo, naturalmente, es imposible explicar detalladamente todos los verbos. Por lo tanto, hay que considerar cómo distribuir el tiempo. En función de esto, a los verbos más difíciles se les asigna más tiempo, mientras que los verbos fáciles de aprender quizá no exigen tanta dedicación y basta con repartir algún material de resumen acerca de sus usos.

Pero, ¿cuáles son los verbos difíciles y cuáles son los fáciles? Para resolver esta cuestión, desarrollamos un método con el que medir el grado de dificultad de aprendizaje de los 50 verbos que trataremos en el curso. Dicha evaluación posibilita vislumbrar con claridad a qué verbos debemos dedicar mayor tiempo y cuidado, pero también los motivos que los hacen complicados para nuestros estudiantes japoneses. Así,

\footnotetext{
${ }^{1}$ Entendemos colocaciones como combinaciones frecuentes en la relación semántica. Para más detalles, véase Bosque (2001) y Koike (2001).

${ }^{2}$ En Japón esta estrategia de ampliar el vocabulario se aplica en varios materiales de inglés como Barnard (2010)
} 
este índice facilita una planificación de la enseñanza más eficaz y práctica.

\subsection{Estado de la cuestión -dificultad de las palabras-}

La novedad de este estudio está en el hecho de medir y cuantificar la dificultad léxica. En la tradición de la lingüística, especialmente de la lingüística aplicada y psicolingüística inglesa, el concepto de la dificultad léxica se ha trabajado en varios estudios, pero nótese que el motivo era distinto que el nuestro y sin intención de cuantificarlo y establecer un ranking de la dificultad de las palabras. Por ejemplo, muchos de los estudios de la lingüística inglesa que trabaja la dificultad de las palabras son del ámbito del procesamiento de oraciones (Rayner \& Duffy 1986, Gennari \& Poeppel 2003, etc.). El interés de este tipo de trabajos radica en aclarar cómo afecta la complejidad léxica a los procesos de lectura.

En el área de la enseñanza y adquisición de la lengua, sí se trabajó el concepto, como en Laufer (1989 y 1990), pero a un nivel meramente teórico. Es decir, el objetivo de los estudios en esta línea es contemplar cuáles son los factores que afectan a la dificultad de las palabras, sin que exista un trabajo que comparta la experiencia de medir la dificultad de las palabras y aplicar el conocimiento conseguido a las clases, que es la característica distintiva de nuestro trabajo.

En las próximas secciones discutiremos qué elementos afectan a la dificultad de aprendizaje y cómo cuantificarlos.

\section{Dificultad de aprender verbos}

Tanto estudiantes como docentes albergan, consciente o inconscientemente, la idea de que entre los verbos hay algunos que resultan difíciles de aprender y otros que se aprenden con mayor facilidad. Por ejemplo, la mayoría de los hispanohablantes y estudiantes de español piensan o intuyen, aunque no puedan explicar el porqué, que manejar el verbo dar resulta mucho más difícil que usar el verbo estudiar. Pero ¿cómo surge esta diferencia en la dificultad de aprendizaje? ¿De qué depende esa dificultad?

Nosotros consideramos que la explicación recae en tres elementos principales, que son los siguientes: número de sentidos que tiene cada verbo, distribución de frecuencia de cada sentido y volumen de la correspondencia de los verbos en español con la lengua materna de los estudiantes.

Primero, naturalmente, los verbos polisémicos son más difíciles de aprender que los verbos que tienen pocos sentidos. Por ejemplo, los verbos dar y estudiar, a los que ya nos hemos referido, difieren mucho en el número de significados que tienen (según DRAE, dar presenta 53 sentidos diferentes, pero estudiar, en la práctica, únicamente $5 .^{3}$ La polisemia del verbo dar obviamente genera dificultad para los estudiantes.

Aparte, el porcentaje de frecuencia de cada significado tiene mucho que ver con la dificultad del aprendizaje. Veamos, a modo de ejemplo, los verbos enviar y permitir, ambos con dos sentidos, pero con una distribución entre ellos totalmente distinta. Las

\footnotetext{
${ }^{3}$ En DRAE se presentan 7 sentidos del verbo estudiar, pero dos de ellos son catalogables de "poco usados" o "en desuso".
} 
definiciones ${ }^{4}$ de enviar son: (1) «Encomendar a alguien que vaya a alguna parte» cuando sus objetos directos denotan personas o grupos de personas, mientras que, cuando los objetos son entidades inanimadas, el verbo denota (2) «Hacer que algo se dirija o sea llevado a alguna parte». En el caso de permitir $^{5}$ : (1) «Dicho de quien tiene autoridad competente: Dar su consentimiento para que otros hagan o dejen de hacer algo» y (2) «Hacer posible algo». Según nuestro análisis en European Spanish Web Corpus, un $0.85 \%$ de los objetos asociados al verbo enviar denotan personas y el resto alude a cosas inanimadas. Así, el sentido (1) es muy infrecuente, lo que convierte a este verbo en prácticamente monosémico. En cambio, según el análisis, en un $45 \%$ de los ejemplos de permitir, el verbo se usa en el sentido (1). Por su parte, el 55\% restante indica el sentido (2), por lo que la distribución de ambas acepciones del verbo permitir están bastante equilibradas. Es, por lo tanto, un verbo verdaderamente bisémico, lo que hace más difícil su asimilación y uso por parte de los estudiantes, o no nativos, que en el caso de enviar.

Igualmente, consideramos que el volumen de la correspondencia entre español y la lengua materna de los estudiantes también puede variar la dificultad. Por ejemplo, para los estudiantes cuya lengua materna es inglés, entender el uso de dar no será especialmente complicado porque el verbo suele traducirse al inglés give en muchos contextos. En cambio, para los estudiantes japoneses es mucho más difícil entender el uso de dar por la falta de la correspondencia con el idioma japonés. El sentido más frecuente del verbo dar es la acción de transferencia, donación, por lo tanto, suele considerarse que el verbo correspondiente de dar en japonés es ataeru, que denota la misma acción. No obstante, el verbo dar tiene muchos más sentidos y usos aparte de denotar acciones de transferir algo, mientras que el verbo ataeru solo denota la acción y no se usa como un verbo de apoyo ni se combina con sustantivos que denotan sentimientos como dar: me da rabia/*ikari wo watashi ni ataeru (lit. me da rabia). Así, en japonés no existe un verbo que generalmente se corresponda con dar y esta falta de correspondencia causa dificultad a los nativos de dicho idioma. En términos generales, el grado de la correspondencia también puede afectar la dificultad del aprendizaje.

Medimos la dificultad de aprendizaje evaluando estos tres factores de los verbos.

Aparte de estos factores, hay otros que pueden afectar la dificultad, como la presencia y la frecuencia de usos gramaticales. Por ejemplo, uno de los usos más importantes de llevar es la combinación con los gerundios; Llevo estudiando español 3 años. El verbo levantar, se usa en la forma reflexiva más que otros verbos; Me levanté a las seis. Estos usos gramaticales son factores que complican el manejo de los verbos. No obstante, de momento, no los tenemos en cuenta puesto que nos falta todavía desarrollar el método de evaluar cómo complican el aprendizaje de los verbos. Por lo tanto, hablando más estrictamente, nuestro índice mide la dificultad léxica de aprendizaje de los verbos.

\section{Evaluación de la dificultad de aprendizaje}

Teniendo en cuenta que la dificultad de aprendizaje de los verbos transitivos se ve afectada por los tres elementos presentados en el apartado 2., puntuamos los 50 verbos

\footnotetext{
${ }^{4}$ Siempre según DRAE.

${ }^{5}$ En DRAE se presentan 4 usos para el verbo transitivo permitir pero según nuestra metodología, que presentaremos en la próxima sección, los 4 usos se clasifican en dos tipos.
} 
transitivos más frecuentes utilizando European Spanish Web Corpus. La evaluación consecuente sirve como brújula a la hora de organizar el curso. En las subsecciones que siguen presentamos el método de evaluación de la dificultad.

\subsection{Recogida de colocaciones}

Primero, para medir el número de significados que tiene cada verbo y especificar su distribución de frecuencia, reunimos los 100 objetos directos más frecuentes para cada verbo. Es decir, reunimos las 100 colocaciones más usadas de tipo «V $+\mathrm{N} »$ formadas por cada uno de los verbos que intentamos evaluar. Evaluamos la dificultad por analizar estas colocaciones.

Para la recogida de colocaciones, utilizamos European Spanish Web Corpus. Este corpus tiene una función que se llama word sketch. Utilizándola se obtienen las listas de los sustantivos que coaparecen más frecuentemente con los verbos en cuestión, la frecuencia de coocurrencias y el $\log$ Dice score $^{6}$ de cada combinación. Por ejemplo, en el caso de dar, consultándolo en el corpus, se obtienen los datos que refleja la tabla siguiente:

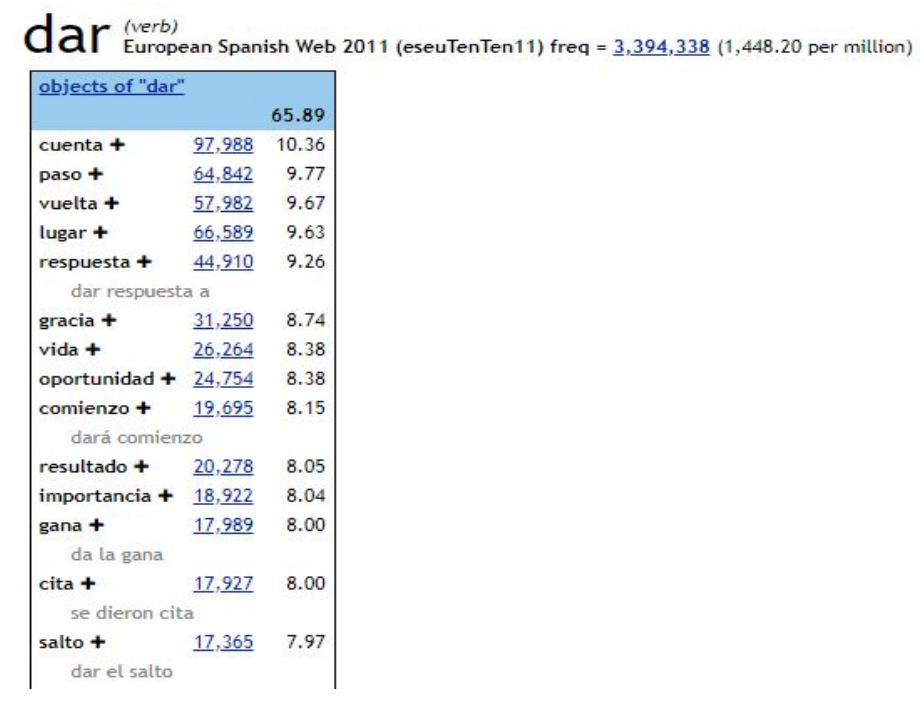

Tabla 1. Un análisis de co-ocurrencias más frecuentes de dar

\subsection{Medida de dificultad}

Como hemos mostrado ya, el sentido de los verbos transitivos depende principalmente de los tipos semánticos de sus objetos. En el caso de dar, por ejemplo, si los objetos son entidades concretas, el verbo se entiende que denota la acción de transferir y donar algo. Si los objetos son de tipo sustantivos eventivos, el verbo tiene que ser usado como verbo de apoyo, como en dar una vuelta. Así, se sabe en qué sentido se usa un verbo transitivo por observación de sus objetos. Aprovechando esta característica, analizamos las colocaciones obtenidas y clasificamos el sentido de los verbos en cada combinación.

\footnotetext{
${ }^{6} \log$ Dice es un índice que representa la fuerza de vinculación de colocaciones desarrollado en Rychly (2008). Cuanto más alta es la puntuación, más estrechamente vinculadas están las palabras. El valor máximo del índice es 14 y puede llegar bajo cero.
} 
Empleamos el diccionario Shogakukan seiwachu jiten 'Diccionario Shogakukan español-japonés' para la clasificación. El motivo de clasificar los significados según su correspondencia con el japonés es la necesidad de tener en cuenta las discrepancias que hay entre ambos idiomas. En el caso de dar, su significado en combinación con cada objeto directo se distribuye tal como se muestra en la tabla siguiente:

\begin{tabular}{|l|r|l|}
\hline ob jetos & frecuenc ia & s ign ificado, uso \\
\hline cuenta & 97,988 & 熟語 'locución' \\
\hline paso & 64,842 & 軽動詞 'verbo de ap oyo' \\
\hline vue Ita & 57,982 & 軽動詞 'verbo de apoyo' \\
\hline lugar & 66,589 & 熟語 'locución' \\
\hline respuesta & 44,910 & 軽動詞 'verbo de ap oyo' \\
\hline oportun idad & 24,754 & 与える 'donar' \\
\hline
\end{tabular}

Tabla. 2. Análisis de significado de dar en combinación con cada objeto frecuente

Después de la clasificación, sumamos el valor de frecuencia de cada significado. A continuación, los dividimos por el valor total de frecuencia respecto de las 100 colocaciones y al final los multiplicamos por 100. Así se sabe en qué porcentaje se usa cada sentido de los verbos. Por ejemplo, el resultado del análisis hasta este punto del verbo dar se resume de este modo:

\begin{tabular}{|l|r|r|}
\hline significado, uso & frecuencia & porcentaje (\%) \\
\hline donar & 480,219 & 42.42 \\
\hline verbo de apoyo & 282,695 & 24.97 \\
\hline locución & 205,596 & 18.16 \\
\hline setimiento & 121,524 & 10.73 \\
\hline estado local & 41,963 & 3.7 \\
\hline total & $1,131,997$ & 100
\end{tabular}

Tabla $3^{7}$. Porcentaje de los significados de dar

Con estos datos calculamos el índice que muestra la dificultad de aprendizaje. El cálculo es bastante simple: dividimos el valor porcentual mayor entre el número de significados:

$$
\frac{\text { porcentaje del significado más frecuente }}{\text { número de significados }}
$$

En el caso de dar, el mayor porcentaje, o el significado más frecuente, corresponde a «donar», con un $42.42 \%$, y este verbo tiene 5 tipos de significados y usos («donar», «verbo de apoyo», «locución», «sentimiento» y «estado local»). Por lo tanto, el cálculo que realizamos es dividir 42.42 entre 5 . La cifra resultante, 8.48 , es el número que muestra la dificultad de aprendizaje del verbo dar.

\footnotetext{
${ }^{7}$ Los números en la tabla pueden contener hasta dos decimales.
} 
El motivo de centrarnos en el valor porcentual mayor es que este número está directamente relacionado con la dificultad de aprendizaje, del mismo modo en que el número de significados afecta mucho a la dificultad. Como presentamos en la sección 2 , cuanto más equilibrada está la frecuencia de cada significado más complicado es manejar ese verbo. Recuérdese que la frecuencia de «el significado A» del verbo enviar era $99.15 \%$ y el de B, $0.85 \%$. En cambio, el porcentaje de aparición de un significado del verbo permitir era $55 \%$ y el del otro significado era $45 \%$, por lo que concluimos que permitir es más difícil de aprender que enviar. Así, el porcentaje de cada significado afecta directamente a la dificultad de aprendizaje y lo incluimos en la evaluación. Generalizando más, cuanto menor es el porcentaje del significado más frecuente, más complicado resulta aprender el verbo. La razón por la que desestimamos el porcentaje de significado menos frecuente es por la disponibilidad y comodidad. Este porcentaje es muy pequeño a veces (inferior al $1 \%$ ) y si lo incluyéramos entre los elementos de puntuación, el índice sería demasiado complejo.

Puntuando 50 de los verbos que íbamos a tratar en la clase, al final, se consigue un ranking de la dificultad.

\begin{tabular}{|c|c|c|c|c|c|}
\hline ranking & verbo & puntuación & ranking & verbo & puntuación \\
\hline 1 & tom ar & 2.21 & 26 & resolver & 41.16 \\
\hline 2 & poner & 2.77 & 27 & esperar & 42.29 \\
\hline 3 & sacar & 4.63 & 28 & contar & 42.74 \\
\hline 4 & Ilevar & 5 & 29 & sentir & 43.29 \\
\hline 5 & hacer & 7.2 & 30 & com er & 47.25 \\
\hline 6 & segu ir & 7.34 & 31 & ob tener & 47.51 \\
\hline 7 & dar & 8.48 & 32 & perder & 47.61 \\
\hline 8 & jugar & 8.6 & 33 & m e jorar & 48.64 \\
\hline 9 & ganar & 10.05 & 34 & escrib ir & 49.07 \\
\hline 10 & trab a jar & 14.67 & 35 & usar & 49.58 \\
\hline 11 & tener & 14.77 & 36 & pagar & 49.69 \\
\hline 12 & pasar & 14.99 & 37 & hab lar & 100 \\
\hline 13 & cum $\mathrm{p}$ lir & 15.4 & 38 & leer & 100 \\
\hline 14 & ver & 18.73 & 39 & rea lizar & 100 \\
\hline 15 & tratar & 23.07 & 40 & $m$ antener & 100 \\
\hline 16 & dec ir & 25.85 & 41 & buscar & 100 \\
\hline 17 & perm itir & 27.17 & 42 & crear & 100 \\
\hline 18 & expresar & 28.55 & 43 & evitar & 100 \\
\hline 19 & saber & 29.51 & 44 & cam b iar & 100 \\
\hline 20 & de jar & 29.72 & 45 & usar & 100 \\
\hline 21 & rec ib ir & 30.73 & 46 & entender & 100 \\
\hline 22 & querer & 33.5 & 47 & lograr & 100 \\
\hline 23 & vivir & 35.26 & 48 & com partir & 100 \\
\hline 24 & consegu ir & 35.99 & 49 & u tilizar & 100 \\
\hline 25 & defender & 38.25 & 50 & com prar & 100 \\
\hline
\end{tabular}

Tabla 4. Un ranking de dificultad de aprendizaje de los verbos

Cómo hemos anticipado, cuanto menor es el índice, más difícil es el verbo. Por lo tanto, según nuestro análisis, los verbos tomar, poner y sacar son los verbos más difíciles para los nativos de japonés ${ }^{8}$. El número máximo del índice es 100 y los verbos de esta

${ }^{8}$ El verbo tomar resultó ser el más difícil por tener abundantes sentidos y usos (10 tipos) y por el hecho de que sus frecuencias estén bien equilibradas (el significado más frecuente «coger» se observó solo en $23.9 \%$ de los ejemplos). A través del análisis de poner, se confirmó que este verbo tiene más tipos de 
puntuación son los más fáciles y monosémicos.

\section{Aplicar datos a la clase}

Las puntuaciones presentadas en la tabla 4 nos indican a qué verbos debemos dedicar el mayor tiempo y esfuerzo en la clase. Así, podemos hacer la clase más eficaz y práctica. Además, al puntuar los verbos, nos enteramos de cuáles son los objetos más frecuentes de cada uno de ellos. Aprovechando los resultados del análisis, desarrollamos un material didáctico de los verbos y sus colocaciones. Por ejemplo, la figura siguiente es una muestra del material que hicimos para enseñar los usos de dar. Utilizando este material, enseñamos los usos típicos de los verbos.

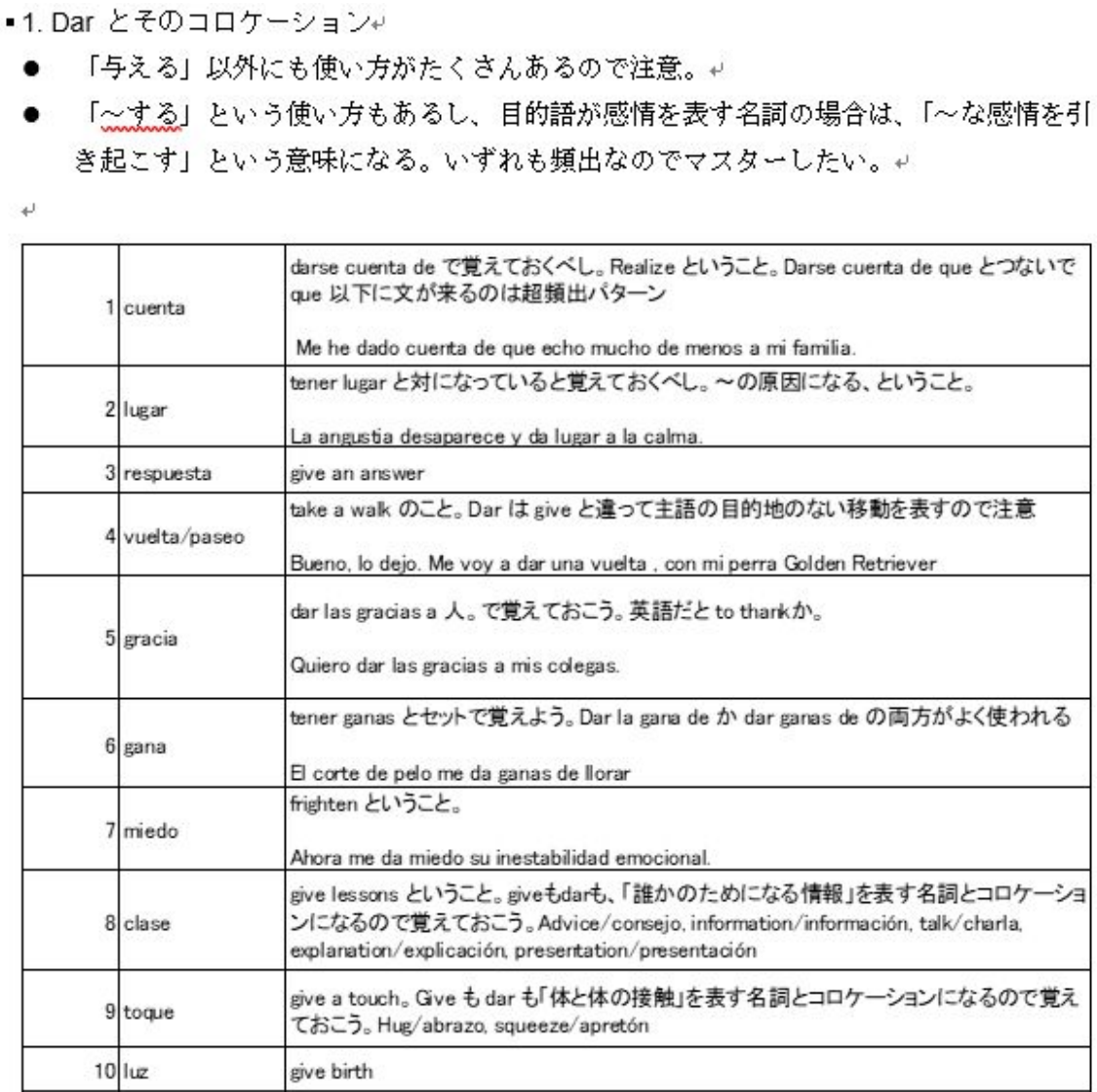

Imagen 1. Una muestra de nuestro material basado en el índice

Aparte de estas ventajas ya detalladas sobre el uso del índice, los datos obtenidos en el proceso también son de gran utilidad en las clases. Veamos cómo:

Primero, especificamos el porcentaje de las frecuencias de significados de cada verbo como hicimos en la tabla 3. Estas tablas, que presentamos directamente en la clase a los estudiantes, muestran las situaciones reales de los usos de nuestros verbos. Es decir,

significados y usos que tomar, pero la distribución de frecuencia de estos no es tan equilibrada como la de tomar. Por esta razón este verbo sigue a tomar. La frecuencia de los significados y usos de sacar se distribuye equilibradamente. Hasta el significado más frecuente «conseguir» se observa solo en $27.8 \%$ de los ejemplos. Por eso se sitúa en el tercer puesto. 
sirven para profundizar en la polisemia de los verbos desde la perspectiva de la frecuencia. Además, las tablas también contribuyen a eliminar el prejuicio, que suelen tener los estudiantes de nivel inicial, de que «a una palabra le corresponde un significado».

Segundo, la frecuencia de las colocaciones que sacamos del corpus es otro factor de utilidad. En una clase, tratamos unas 20-35 colocaciones y todas son las que aparecen con gran frecuencia. En otros términos, apoyándonos en los datos que reunimos, podemos presentar solo las colocaciones que son realmente frecuentes. En estos datos se observan discrepancias entre las situaciones reales de los usos de los verbos y las explicaciones que tradicionalmente reciben los estudiantes de español. Por ejemplo, según los diccionarios presentados arriba, DRAE y SSJ, el significado primario del verbo seguir es «ir detrás de alguien» pero en el corpus solo en los $9 \%$ de los ejemplos de «seguir $+\mathrm{N}$ » se observa seguir en ese sentido y confirmamos que en la gran mayoría de los casos el verbo se usa en el sentido de «respetar la regla» como en Siga las instrucciones que le indicó su dermatólogo. Por lo tanto, se revela que es más práctico y lógico presentar el verbo en función de esa acepción y así lo hacemos en la clase. El verbo contar, por ser cognado del verbo inglés count, suele considerarse que su significado primario es «numerar, computar» pero según nuestras observaciones, el verbo se usa en el sentido de «referir, relatar» mucho más frecuentemente (14\% vs. $86 \%$ ). Por los datos se sabe cuáles son los significados verdaderamente principales o útiles de cada verbo, contribuyendo a la renovación de los métodos docentes.

A través del análisis observamos muchas colocaciones que se usan frecuentemente pero casi nunca se presentan en los libros de texto para los estudiantes. Por ejemplo, el sustantivo que coaparece con hacer como su objeto directo con mayor frecuencia es referencia, sin embargo, esta colocación no suele presentarse en los materiales ${ }^{9}$. Cuando el significado del sustantivo es estudioso o de especialidad, las colocaciones que incluyen tales tipos de sustantivos se descartan. Sacar partido, tener derecho, pagar cuota/multa, alcanzar acuerdo son colocaciones muy frecuentes. Aun así, quizás por la formalidad de los sustantivos, no se tratan en los libros docentes. Sin embargo, según nuestra opinión, colocaciones de estos tipos son las más vivas y merecen atención en la clase. Estos tipos de colocaciones pueden ser confusos para los estudiantes de menor edad, pero en nuestro caso se trata de universitarios. Además, en nuestra universidad, las clases de español se imparten pensando en capacitar a los alumnos para la lectura, especialmente de literatura académica, de artículos de sus especializaciones. Por lo tanto, tratamos las colocaciones de carácter formal si las vemos útiles y frecuentes.

\section{Conclusión y trabajos en futuro}

En este trabajo presentamos nuestra experiencia de impartir clases acerca de los significados y usos de los verbos más frecuentes. La mayor parte del discurso de los nativos se construye mediante las 600 palabras más frecuentes y estas palabras, por ser altamente polisémicas o tener muchos usos, suelen ser complicadas para los estudiantes. De hecho, consideramos sumamente útil enseñar cómo se usan tales palabras. Nos centramos en los verbos y analizamos la dificultad de su aprendizaje. El resultado del

\footnotetext{
${ }^{9}$ En la Universidad de Kyushu, se usan dos materiales en las clases de los estudiantes de primer grado y en ambos textos no se presenta la colocación hacer referencia.
} 
análisis pone de relieve cuáles son los verbos que necesitan una explicación detallada y cuáles son los significados y usos que debemos tratar en la clase. Sin embargo, nuestro análisis tiene su límite y solucionarlo es nuestro trabajo en el futuro. Primero, para puntuar la dificultad, enfocamos los objetos directos de los verbos, por lo tanto, lógicamente, de momento es imposible puntuar los verbos intransitivos. Evaluarlos junto con los verbos transitivos requiere desarrollar un método de evaluación distinto.

Segundo, como hemos indicado en la sección 2, consideramos solamente las diversidades léxicas de los verbos y descartamos sus comportamientos sintácticos en la evaluación. Incluir estas perspectivas en la puntuación, permitiría precisar más la evaluación y los significados y usos que son verdaderamente útiles.

\section{Bibliografía}

BARNARD, Cristopher (2010). 100 no cho-kihon meishi de hirogaru eigo collocation 'Ampliar colocaciones con los 100 sustantivos más frecuetes en inglés'. Tokio: Place.

BOSQUE MuÑOZ, Ignacio (2001). «Sobre el concepto de 'colocación' y sus límites». Lingüistica Española Actual, 23 (1), 9-40.

GENNARI, Silvia, \& POEPPEL, David (2003). «Processing correlates of lexical semantic complexity». Cognition, 89 (1), 27-41.

LAUFER, Batia (1989). "A factor of difficulty in vocabulary learning: Deceptive transparency». AILA review, 6 (1), 10-20.

LAUFER, Batia (1990). "Why are some words more difficult than others? - Some intralexical factors that affect the learning of words». International Review of Applied Linguistics in Language Teaching, 28 (4), 293-308.

KoIKe, Kazumi (2001). Colocaciones léxicas en el español actual: estudio formal y léxico-semántico. Madrid/Tokio: Universidad de Alcalá/Universidad de Takushoku, 2001.

RAYNER, Keith \& DUFFY, Susan (1986). «Lexical complexity and fixation times in reading: Effects of word frequency, verb complexity, and lexical ambiguity». Memory \& cognition, 14 (3), 191-201.

Real ACADEmia EsPañola (2014). Diccionario de la lengua española, $23^{\text {a }}$ edición. Madrid: Espasa. (DRAE).

RYCHLÝ, Pavel (2008). «A lexicographer-friendly association score». Proceedings of Recent Advances in Slavonic Natural Language Processing, 6-9.

SketCH ENGINE (2011). European Spanish Web Corpus [2011, Eu + Am, Freeling v4] (9,497,402,122 palabras). Recuperado el 25/10/17, de https://the.sketchengine.co.uk/

TAKAGAKI, Toshihiro (dir) (2007). Shogakukan seiwachu jiten, Segunda edición. Tokio: Shogakukan. (SSJ) 